\title{
First experience of $\alpha 1,3$-galactosyltransferase gene-knockout (GTKO) transgenic pig to nonhuman primate lamellar corneal xenotransplantation
}

\author{
Ik Jin Yun ${ }^{1}$, Ki Cheul Shin ${ }^{2}$, Hye Sun Shin ${ }^{1}$, Wan Seop Kim ${ }^{3}$, Madhuri Saindane ${ }^{1}$, Keon Bong $\mathrm{Oh}^{4}$, Hee Jung Kang ${ }^{5}$, \\ Yu Rim Ahn
}

\author{
${ }^{1}$ Department of Surgery, Konkuk University Medical Center, Seoul, Korea \\ ${ }^{2}$ Department of Ophthalmology, Konkuk University Medical Center, Seoul, Korea \\ ${ }^{3}$ Department of Pathology, Konkuk University Medical Center, Seoul, Korea \\ ${ }^{4}$ Division of Animal Biotechnology, National Institute of Animal Science, Wanju, Korea \\ ${ }^{5}$ Department of Laboratory Medicine, Hallym University College of Medicine, Chuncheon, Korea
}

Background: Corneal allotransplantation is a well-known technique to treat corneal blindness. However, the problem of organ scarcity in transplantology has become profound. One promising alternative could be xenograft using pig as an organ donor. Full thickness corneal xenotransplantation needs total immunosuppression. This study is an investigation of the efficacy of a1,3-galactosyltransferase gene-knockout (GTKO) transgenic pig-to-nonhuman primate lamellar corneal transplantation with minimal immunosuppression.

Methods: We conducted 10 lamellae corneal xenotransplantation between 2016 and 2019. Clinically acceptable graft size (diameter $7.5 \mathrm{~mm}$, thickness $500 \mathrm{um})$. The dexamethasone subconjunctival injection $(1.5 \mathrm{mg} / 0.3 \mathrm{~mL})$ was administered for minimal immunosuppression immediately after surgery and eye drops of $0.5 \%$ levofloxacin and $1 \%$ prednisolone acetate were applied four times a day for 1 week, gradually tapered. No eye drops were added after two months. Three cases are alive without graft rejection. We examined the remaining seven cases for the histopathological features and Immunologic profiles.

Results: As a result, three of the ten xenografts survival is significantly longer $1,239,589$, and 316 days. Corneal opacity resulted in graft failure, and terminated in seven cases. Rejected grafts showed extensive polymorphic cellular infiltration, different degrees of epithelial layer irregular attenuation, stromal neovascularization, and inflammatory cell infiltrations including lymphocytes, plasma cells, eosinophils, neutrophils. Stromal irregularity, fibrosis and edema are observed in two of seven cases resulting in a single case of sub epithelial detachment. Immunologic profiles of the recipients with rejected grafts shows minimal increase in anti-Gal antibody IgG and IgM but increase in anti-Gal IgG is seen in one case. Four cases have different systemic inflammatory conditions with regards to plasma C3a and D-dimer levels. The anterior stromal surface of the graft showed epithelial nests and fibrous proliferation.

Conclusions: The GTKO transgenic pig to NHP lamella xeno corneal transplantation could be a promising substitute for human corneal allograft. Lamella xeno corneal transplantation may be a feasible option with minimal immunosuppression.

Corresponding author: Ik Jin Yun

E-mail: ijyun@kuh.ac.kr

\section{(c) The Korean Society for Transplantation}

This is an Open Access article distributed under the terms of the Creative Commons Attribution Non-Commercial License (http://creativecommons.org/licenses/by-nc/4.0/) which permits unrestricted non-commercial use, distribution, and reproduction in any medium, provided the original work is properly cited. 